\title{
Venous thromboembolism following ankle arthroscopy
}

\begin{abstract}
Background: The number of ankle arthroscopies being performed is increasing for both diagnosis and treatment of intra-articular pathology. Venous thromboembolism is an uncommon complication following ankle arthroscopy, but can have devastating outcomes. Publication of procedure-specific studies evaluating rates of post-operative thromboembolism is lacking, and current guidelines reflect this.
\end{abstract}

Aim: To evaluate the incidence of thromboembolism in a consecutive series of patients undergoing diagnostic ankle arthroscopy, with intervention requiring immobilisation in a non-weight bearing cast post-operatively.

Method: An analysis of consecutive patients undergoing diagnostic ankle arthroscopy with either ligament reconstruction or supramalleolar osteotomy by a single surgeon over a 12 month period. A retrospective review of the incidence of any complications was undertaken, with a particular focus on venous thromboembolism.

Results: 104 patients underwent ankle arthroscopy during the 12 month period. All patients completed routine follow-up at 6 weeks, 3 months and 6 months. The overall complication rate was $3.8 \%$. The incidence of venous thromboembolism in our series was $0.96 \%$.

Conclusion: Our incidence of venous thromboembolism with standard use of low molecular weight heparin in all patients is lower than previously published. Larger trials will aid in identifying whether chemoprophylaxis is required in all those undergoing ankle arthroscopy with post-operative immobilisation, or just patients with additional risk factors.
Volume 3 Issue I - 2015

\author{
Maire-Clare Killen, Prasad Karpe, Rajiv \\ Limaye \\ Orthopaedic Department, University Hospital North Tees, UK
}

Correspondence: Maire-Clare Killen, Orthopaedic

Department, University Hospital North Tees, Hardwick Road,

Stockton-on-Tees, UK, Tel 7736934388,

Email maire-clare.killen@doctors.org.uk

Received: April 12, 2015 | Published: July 02, 2015
Abbreviations: DVT, deep vein thrombosis; PE, pulmonary embolism; NICE, national institute for health and care excellence; BOFAS, British orthopaedic foot and ankle society

\section{Introduction}

Arthroscopy is becoming an increasingly common surgical procedure for diagnosis and treatment of intra-articular ankle pathology. As more arthroscopies are being performed with increasingly complex therapeutic procedures, the potential for complications rises. ${ }^{1}$ Complication rates as a whole published in the literature vary significantly, with the incidence of venous thromboembolism thought to be relatively low. ${ }^{2}$ However, existing evidence is lacking, particularly for procedure-specific rates of thromboembolism. Current guidelines reflect this gap in knowledge and recommend risk assessing individual patients, with use of chemoprophylaxis on a patient-by-patient basis. ${ }^{3,4}$ All surgery of the foot and ankle comes with a theoretical increase in the risk of postoperative thromboembolism and with subsequent immobilisation in a non-weight bearing plaster, this risk increases further. Deep vein thrombosis (DVT) or pulmonary embolism (PE) can result in significant morbidity and possible mortality, therefore it is important to take measures to lower the incidence and improve outcomes. The aim of our study was to evaluate the incidence of thromboembolism in a consecutive series of patients undergoing diagnostic ankle arthroscopy, with intervention requiring immobilisation in a nonweight bearing cast post-operatively.

\section{Materials and methods}

We undertook a retrospective review of consecutive patients undergoing diagnostic ankle arthroscopy with either subsequent ligament reconstruction or supramalleolar osteotomy under the care of the senior author over a 12 month period. All patients undergo diagnostic ankle arthroscopy using anteromedial and anterolateral portals. This is performed to assess intra-articular pathology prior to proceeding with modified Brostrom-Gould lateral ligament reconstruction or supramalleolar osteotomy for deformity correction in osteoarthritis of the ankle.

Following these procedures, patients are initially put into a backslab for 2 weeks, then clinically assessed and put into a non-weight bearing lightweight cast for a further 4 weeks. As routine, patients are continued on prophylactic dose of low molecular weight heparin for 6 weeks, while they are non-weight bearing, which is dosed depending on patient weight. Further clinical assessments occur on an outpatient basis at 2 weeks, 6 weeks, 3, 6 and 12months for any complications. Clinic letters and documentation in the patient notes were reviewed for the occurrence and nature of any post-operative complications. Imaging software was reviewed to ascertain the number of patients undergoing Doppler ultrasound or imaging of the chest for suspected thromboembolic events and the outcome of such studies.

\section{Results}

A total of 104 patients underwent ankle arthroscopy over a 12 month period. Patient demographics and procedures are summarised 
in Table 1. The overall complication rate in our series was 3.8\%. 2 patients had superficial wound infections, which resolved with a short course of oral antibiotics. One patient had a superficial peroneal nerve injury post-operatively. Five patients underwent Doppler ultrasound for calf pain and suspected DVT, with confirmation of a popliteal vein thrombosis in one patient despite prophylactic heparin. No patients underwent imaging for suspected PE.

Table I Patient and procedure demographics

\section{Demographic}

\begin{tabular}{ll}
\hline Mean age & 45.3 \\
(Range ) & $(17-75)$ \\
Male: Female ratio & $01: 01$ \\
Mean BMI & 29.8 \\
(Range) & \\
\hline Procedure & $(23-49)$ \\
\hline
\end{tabular}

Modified Brostrom-Gould \pm Syndesmosis

Reconstruction

89

Modified Brostrom-Gould, Syndesmosis Reconstruction \& Microfracture

4

Modified Brostrom-Gould, Syndesmosis

Reconstruction \& Osteochondral Repair

Multiple Ligament \& Syndesmosis Reconstruction

Supramalleolar Osteotomy

The incidence of venous thromboembolism in our series was $0.96 \%$. One 43 -year old female patient with a body mass index (BMI) of 48 and no other co-morbidities had a popliteal vein thrombosis, diagnosed on Doppler ultrasound 8 weeks post-operatively. She had received a weight-adjusted 6 week course of chemoprophylaxis, which was stopped at 6 weeks post-operatively when her cast was removed and full-weight bearing commenced. Despite this however, she developed calf pain 2 weeks later with confirmation of DVT and was commenced on oral anticoagulation with warfarin. On further questioning, despite being able to fully weight bear, her mobility had not fully returned to her pre-operative state.

\section{Discussion}

Ankle arthroscopy has become the treatment of choice for diagnosis and treatment of the vast majority of intra-articular pathologies. As the complexity of procedures increases, with a longer surgical time, there is theoretically a higher risk of post-operative thromboembolism. ${ }^{1}$ At present, there is no clear published evidence regarding the rate of DVT or PE following ankle arthroscopy with ligament reconstruction or supramalleolar osteotomy, or the use of low molecular weight heparin following such procedures. Existing trials have evaluated the incidence of thromboembolism in patients immobilised in plaster; rates of DVT or PE in such studies range from $4-40 \%$ when chemoprophylaxis is not used. However, these studies are non-specific to individual procedures. ${ }^{3,5-9}$

UK guidelines from the National Institute of Health and Care Excellence (NICE) and the British Orthopaedic Foot and Ankle Society (BOFAS) recommend that patients undergoing foot and ankle surgery are assessed individually, with the use of chemoprophylaxis on a patient-by-patient basis. ${ }^{3,4}$ Patient specific risk factors are well established, but controversy continues to exist with procedurespecific risks; there remains no clear identification of which surgical interventions are "high risk" for post-operative thromboembolism.

NICE has further evaluated the use of chemoprophylaxis in patients immobilised in a below-knee plaster. Their recommendation is again, only using chemoprophylaxis in patients with additional risk factors, summarised in Table 2. This is based on the fact there is little evidence to demonstrate that prolonged immobilisation in a below knee plaster, in the absence of patient related risk factors merits the use of chemoprophylaxis, with a potential huge cost implication of using low molecular weight heparin in all patients. ${ }^{3}$

Table 2Additional risk factors for venous thromboembolism post-operatively ${ }^{3}$

$\begin{array}{lll}\text { a. Age }>60 & \text { - } & \begin{array}{l}\text { Critical Care } \\ \text { Admission }\end{array} \\ \text { b. Dehydration } & \text { - } \begin{array}{l}\text { Known } \\ \text { Thrombophilia }\end{array} \\ \text { C. Obesity }(\mathrm{BMI}>30) & \text { - One or more } \\ \text { d. Personal History or First } & \begin{array}{l}\text { Significant Co- } \\ \text { Degree Relative with Venous }\end{array} & \begin{array}{l}\text { Hormone } \\ \text { Replacement } \\ \text { Therapy }\end{array} \\ \text { e. Ohromboembolism } & \text { - } \begin{array}{l}\text { Varicose Veins } \\ \text { with Phlebitis }\end{array}\end{array}$

In patients with additional risk factors highlighted in Table 2, chemoprophylaxis should definitely be used, unless contraindicated. BOFAS recommends that this should be started 6-12 hours postoperatively and continued until the patient gains adequate mobility; the non-weight bearing plaster is removed, or when weight bearing is permitted. ${ }^{4}$

Despite this existing guidance, the occurrence of post-operative thromboembolism, albeit relatively rare, can result in significant morbidity and mortality. The senior author therefore uses heparin in all patients given their prolonged period of immobilisation in a nonweight bearing plaster. This has resulted in a much lower rate of DVT or PE compared to the $4-40 \%$ previously published in the literature. ${ }^{3,5-9}$

The use of low molecular weight heparin in all patients is not without disadvantages. There is the obvious cost implication, but also side effects, including potential increased risk of bleeding. We did not have any incidences of such adverse events in our cohort, but these should be considered and discussed with patients when commencing treatment.

At present, there is obvious indication for the use of chemoprophylaxis to reduce the risk of thromboembolism in patients with additional risk factors. Further research is needed, ideally procedure-specific randomised controlled trials evaluating the role of 
low molecular weight heparin in low risk patients, to determine the need for routine chemoprophylaxis in this group.

\section{Conclusion}

Our rate of thromboembolic events in our series with standard use of 6 weeks of chemoprophylaxis in all patients was $0.96 \%$, lower than previously published in the literature. Despite this, one patient still developed a deep vein thrombosis 8 weeks post-operatively, which highlights the need for continued assessment of patients, with possible prolonged use of low-molecular weight heparin in those who are particularly high risk, until their mobility returns to normal. There is a clear indication for the use of chemoprophylaxis to reduce the risk of thromboembolism in patients with additional risk factors. Further research is needed to evaluate the role of low molecular weight heparin in low risk patients, to determine the need for routine chemoprophylaxis in this group.

\section{Acknowledgments}

None.

\section{Conflicts of interest}

There was no actual or potential conflict of interest in relation to this submission between authors and no financial sponsorship was received for this article. No benefits in any form have been received or will be received from a commercial party related directly or indirectly to the subject of this article.

\section{References}

1. Deng DF, Hamilton GA, Lee M, et al. Complications associated with foot and ankle arthroscopy. J Foot Ankle Surg. 2012;51(3):281-284.

2. Bushnell BD, Anz AW, Bert JM. Venous thromboembolism in lower extremity arthroscopy. Arthroscopy. 2008;24(5):604-611.

3. National Institute for Health and Care Excellence. Venous thromboembolism: Reducing the risk.

4. British Orthopaedic Foot \& Ankle Association. British Orthopaedic Foot and Ankle Society Guidelines for Venous Thromboembolism Prophylaxis.

5. Kock HJ, Schmit-Neuerburg KP, Hanke J, et al. Thrombo-prophylaxis with low-molecular-weight heparin in outpatients with plaster-cast immobilisation of the leg. Lancet. 1995;346(8973):459-461.

6. Lapidus LJ, Rosfors S, Ponzer S, et al. prolonged thromboprophylaxis with dalteparin after surgical treatment of Achilles tendon rupture: a randomized, placebo-controlled study. J Orthop Trauma. 2007;21(1):5257.

7. Lassen RM, Borris LC, Nakov RL. Use of low-molecular-weight heparin reviparin to prevent deep-vein thrombosis after leg injury requiring immobilization. N Eng J Med. 2002;347(10):726-730.

8. Solis G, Saxby T. Incidence of DVT following surgery of the foot and ankle. Foot Ankle Int. 2002;23(5):411-414.

9. Radl R, Kastner N, Aigner C, et al. Venous thrombosis after hallux valgus surgery. J Bone Joint Surg Am. 2003;85-A(7):1204-1208. 